\title{
Comprehensive identification of sensitive and stable ISFET sensing layer high-k gate based on ISFET/electrolyte models
}

\author{
Ahmed M. Dinar ${ }^{1}$, A.S. Mohd Zain ${ }^{2}$, F. Salehuddin ${ }^{3}$ \\ ${ }^{2,3}$ Faculty of Electronics and Computer Engineering, Universiti Teknikal Malaysia Melaka (UTeM), Malaysia \\ ${ }^{1}$ Computer Engineering, University of Technology, Baghdad, Iraq
}

\begin{abstract}
Article Info
Article history:

Received May 29, 2018

Revised Sep 12, 2018

Accepted Nov 8, 2018

Keywords:

High-k material

ISFET

ISFET/electrolyte models

$\mathrm{pH}$ sensitivity

Surface stability

ABSTRACT

The ISFET sensing membrane is in direct contact with the electrolyte solution, determining the starting sensitivity of these devices. A SiO2 gate dielectric shows a low response sensitivity and poor stability. This paper proposes a comprehensive identification of different high-k materials which can be used for this purpose, rather than SiO2. The Gouy-Chapman and Gouy-Chapman-Stern models were combined with the Site-binding model, based on surface potential sensitivity, to achieve the work objectives. Five materials, namely Al2O3, Ta2O5, Hfo2, Zro2 and SN2O3, which are commonly considered for micro-electronic applications, were compared. This study has identified that Ta2O5 have a high surface potential response at around $59 \mathrm{mV} / \mathrm{pH}$, and also exhibits high stability in different electrolyte concentrations. The models used have been validated with real experimental data, which achieved excellent agreement. The insights gained from this study may be of assistance to determine the suitability of different materials before progressing to expensive real ISFET fabrication.
\end{abstract}

Copyright (C) 2019 Institute of Advanced Engineering and Science. All rights reserved.

\section{Corresponding Author:}

Ahmed M. Dinar,

Faculty of Electronics and Computer Engineering,

Universiti Teknikal Malaysia Melaka (UTeM),

Hang Tuah Jaya, 76100 Durian Tunggal, Melaka, Malaysia.

Email: aealzubydi@gmail.com

\section{INTRODUCTION}

There has been a tremendous convergence in the last decade in chemical sensing applications, with CMOS-based micro-technology playing a crucial role in this field. This has been enabled by the use of solid-state sensors that can be implemented in planar form, and manufactured using CMOS technology to monolithically integrated on a single chip. This technology now provides an opportunity for chemical sensing platforms to leverage semiconductor technology that may offer advantages such as scalability, miniaturisation, fabrication, and integration with intelligent instrumentation. ISFET sensors are the most promising, and may satisfy all these opportunities. The essential property of ISFETs is scalability with the developing semiconductor fabrication. This property provides a continuous trend of sensor minimisation, with resulting advantages for biochemical tests, e.g. fast response and the small volume of analyte solution required.

Due to its promising application in biological, biochemical and medical detection [1]-[5]., ISFET has received much interest since it was first reported by Bergveld in 1972 [6]. Particularly, much effort has been made to investigate $\mathrm{pH}$ sensitive ISFETs with studies on device structures and $\mathrm{pH}$-sensing membranes aimed at improving the sensitivity and stability of ISFETs [7], [8]. It is well known that the gate dielectric is in direct contact with the electrolyte solution, which determines the starting sensitivity of these devices. As the $\mathrm{SiO} 2$ gate dielectric shows a low response sensitivity and poor stability, other inorganic materials such as $\mathrm{Al} 2 \mathrm{O} 3$ [7], [8], Si3N4 [6], [9], Ta2O5 [11], [9], $\mathrm{HfO} 2$ [12]-[15] and $\mathrm{ZrO} 2$ [12]-[14] with their enhanced 
stability and sensitivity have also been investigated. Moreover, the drain-source voltage of an ISFET based on a MOSFET structure also affects ionic charges with a horizontal electric field in the electrolyte solution, resulting in a non-uniform charge distribution at the electrolyte-insulator interface. The operating mechanism of a $\mathrm{pH}$-sensitive ISFET is the change of potential between the electrolyte solution and the gate dielectric surface, thus leading to an increased or decreased output current by the ISFET. Moreover, using of Double Gate MOSFETs by prove the ISFET increase the subthreshold swing in case of different top and bottom gate oxide thicknesses [25], [26].

Until now, different models and adaptations of models have been used to model the pH-change sensitivity in ISFETs, the development of surface potential in the area, and in the further course of a Helmholtz double layer (DL). Louis Georges Gouy in 1910, and David Leonard Chapman in 1913, together noticed that capacitance was not a constant, but depended on the applied potential and the ionic concentration. The Gouy-Chapman model made an important contribution by proposing a diffuse model of the DL. In this model, "the charge distribution of ions as a function of distance from the metal surface allows Maxwell-Boltzmann statistics to be applied". Thus, the electric potential decreases exponentially at distances further away from the surface of the fluid bulk. However, the Gouy-Chapman model fails for highly charged DLs. In 1924, Otto Stern suggested combining the Helmholtz model with the Gouy-Chapman model: In Stern's model, some ions adhere to the electrode as suggested by Helmholtz, giving an internal Stern layer, while some form a Gouy-Chapman diffuse layer. Figure 1 demonstrates schematic representations of electrical double layer structures describing (a) the Helmholtz model, (b) the Gouy-Chapman model and (c) the Gouy-Chapman-Stern model.

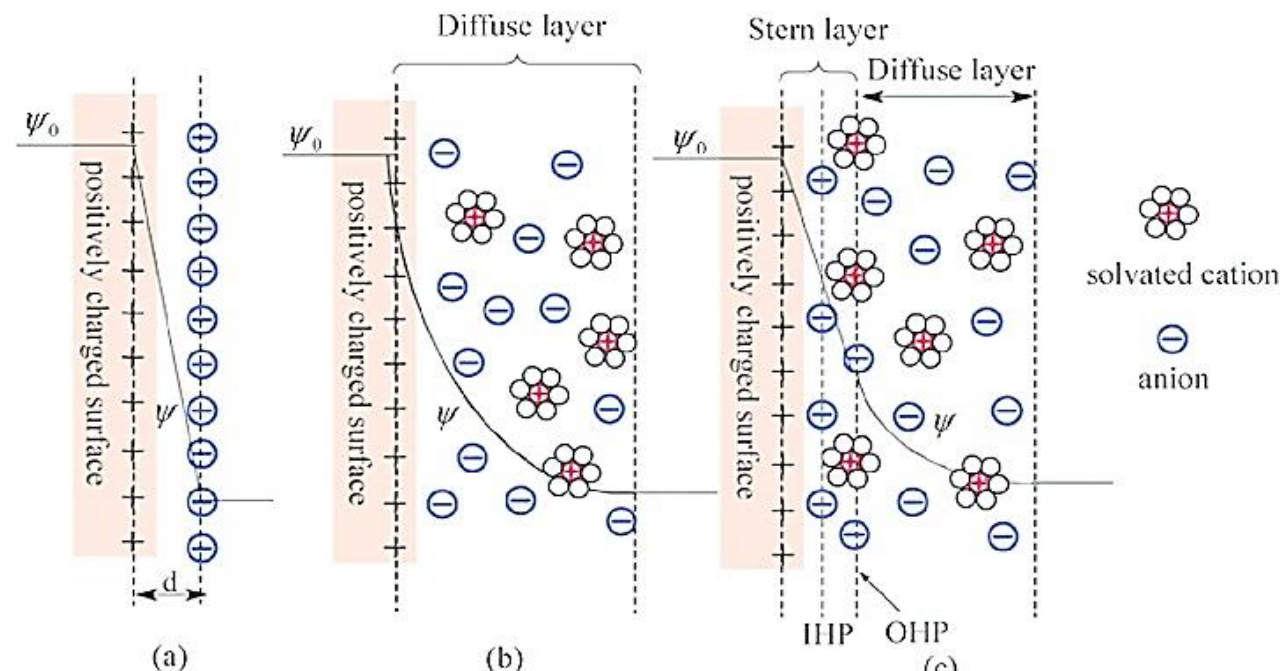

Figure 1. ISFET DL’s models [16]

In this paper, we present a comprehensive identification of high $\mathrm{pH}$-sensitivity insulator layer material (Stern Layer) based on a surface potential sensitivity parameter. The Gouy-Chapman and GouyChapman-Stern models were combined with the Site-binding model to achieve the stated work objectives. The combination of these models will allow for a higher sensitivity using material that is more resistant to changes in electrolyte $\mathrm{pH}$ for ISFET insulator membranes, and furthermore, the identification of the specific capacitance of the Stern layer. Figure 2 below demonstrates the work objective in terms of insulator/electrolyte/semiconductor interfacing ISFETs.

The rest of the sections present the modelling and its validation with experimental real data as reported in Section 2. The model result and a discussion about the ISFET sensitivity of four high-k materials. Are reported in Section 3. Model limitations are mentioned in Section 4 and the conclusions are summarised in Section 5. 


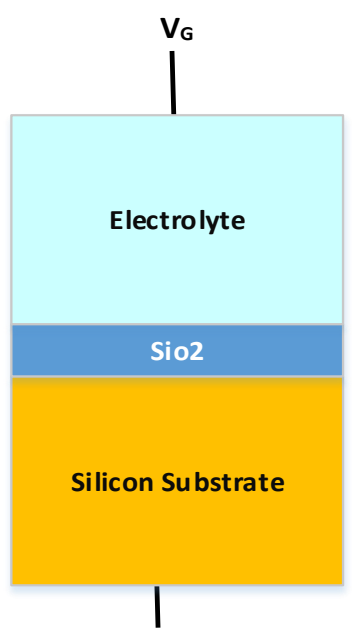

Back Side Gate

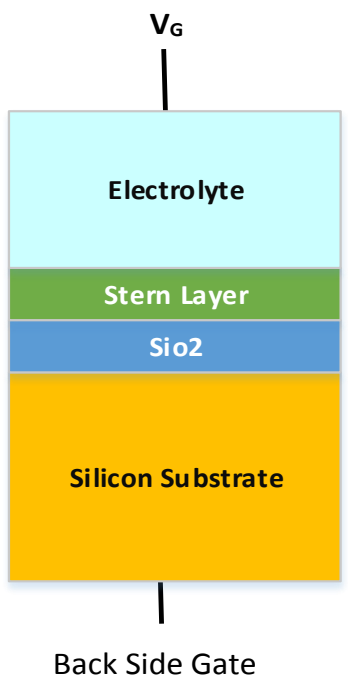

(b)

Figure 2. ISFET structure based on (a) Chapman model (b) Gouy-chapman-stern model

\section{ISFET MODELS AND METHODS}

\subsection{ISFET modelling}

The ISFET has been used for many years to measure the $\mathrm{pH}$ value of electrolyte solutions [17]-[21]. In the original structure of an ISFET, the gate oxide is in direct contact with the electrolyte solution as shown in Figure 2(a), acting as a sensing dielectric. The Stern layer is a modified version of the ISFET in which the sensing layer is separated from the gate oxide by using an extended conductive layer Figure $2 \mathrm{~b}$ after the gate oxide is covered by the electrolyte solution, creating a more robust structure for extended efficacy in the solutions. The area of the sensing gate, compared to the area of the entire sensor, is one of the structural design parameters that greatly influence the electrical behaviour of the sensor, due to the existence of parasitic capacitance which degrades the measured sensitivity of the sensor. The build-up of a surface charge due to protonation/deprotonation reactions induces a potential at the sensor surface. Through the effective coupling capacitance between the sensor surface and Floating Gate (FG), the surface potential $(\psi o)$ modulates the potential of the FG, and therefore there will be a corresponding shift in the threshold voltage (VT) of the sensor [22], [23]. Therefore, from the Site-binding model, the charge density can be expressed by [24]:

$$
\sigma_{0}=q N_{s i l}\left(\frac{a H_{s}^{+2}-K_{a} K_{b}}{K_{a} K_{b}+K_{b} a H_{s}^{+}+a H_{s}^{+2}}\right)
$$

Where; $a H_{s}^{+}$is the $\mathrm{H}+$ activity calculated by $\left(a H_{s}^{+}=10^{-p H s}\right)$; $\mathrm{q}$ is the elementary charge; Nsil is the density of the available sites and $\mathrm{Ka}$; $\mathrm{Kb}$ represents the intrinsic dissociation constants, and the Nsil, $\mathrm{Ka}$ and $\mathrm{Kb}$, are oxide layer dependent.

Based on "charge density", the charge on the electrolyte side of a double layer $(\sigma D L)$ is the same value, but is a negative charge. Therefore, this charge can be calculated from the integral double layer capacitance (Ci) and the surface potential [23]:

$$
\sigma \mathrm{DL}=-\mathrm{C}_{\mathrm{i}} \psi_{\circ}=-\sigma_{\circ}
$$

Therefore, by solving (1) and (2), we can demonstrate the relation between $a H_{s}^{+}$and $\psi o$ parameters.

According to the Boltzmann distribution model for the $\mathrm{H}+$ ions [8], [23], [24], the $\mathrm{pH}$ value at the sensor surface is[8]:

$$
\mathrm{pH}_{\mathrm{S}}=\mathrm{pH}_{\mathrm{B}}+\frac{\mathrm{q} \Psi_{0}}{2.3 \mathrm{kT}}
$$

$\mathrm{T}$ and $\mathrm{k}$ represent the absolute temperature and Boltzmann constant, respectively. The subscript $\mathrm{S}$ and $\mathrm{B}$ denote the $\mathrm{pH}$ at the sensor surface and in the bulk solution, respectively, and $\psi \mathrm{o}$ is the potential drop 
across the diffusion layer. In Equation (3), the intrinsic buffer capacity ( $\beta$ int) is defined as the ability to collect a charge at the sensor surface $(\sigma 0)$ due to the change in surface $\mathrm{pH}(\mathrm{pHs})[8]$ :

$$
\beta_{\text {int }}=\frac{\Delta \sigma_{0}}{-\mathrm{q} \Delta \mathrm{pH}_{\mathrm{S}}}
$$

The diffusion capacity (Cdiff) is the ability to store the opposing charge in the solution near the surface, due to the change in surface potential [8]:

$$
\mathrm{C}_{\text {diff }}=\frac{\Delta \sigma_{0}}{\Delta \psi_{0}}
$$

Therefore, we can write:

$$
\frac{\Delta \psi_{0}}{\Delta p H_{S}}=-q \frac{\beta_{\text {int }}}{C_{\text {diff }}}
$$

Differentiating Equation (3) with respect to the bulk pH, and using Equation (6), the surface potential sensitivity to the $\mathrm{pHB}$ can be derived as [9].

$$
\frac{\Delta \Psi_{0}}{\Delta \mathrm{pH}_{\mathrm{B}}}=-2.3 \frac{\mathrm{kT}}{\mathrm{q}}\left(\frac{1}{1+\alpha}\right)
$$

With,

$$
\alpha=\frac{2.3 k T C_{\text {diff }}}{q^{2} \beta_{\text {int }}}
$$

Here, $\alpha$ is a positive sensitivity (dimensionless parameter). Therefore, the sensitivity of the potential at the sensor surface and the corresponding change of the sensor threshold voltage to the bulk pH are limited to $59.3 \mathrm{mV} / \mathrm{pH}$ (Nernst limit).

In the Stern model, the relationship between the diffusion layer potential $\psi$ o (previous calculations) and the Stern potential $\psi$ s can be expressed by:

$$
(\psi o-\psi s)=\frac{\left(8 k T \varepsilon \varepsilon^{\circ} n^{o}\right)^{\frac{1}{2}} \sinh \left(\frac{z q \psi s}{2 k T}\right)}{C_{S}}
$$

Where $\varepsilon \varepsilon_{\circ}$ is the permittivity of free space and it relative permittivity, respectively; $n^{o}$ is the number concentration of each ion in the bulk, $\mathrm{z}$ is the magnitude of the charge on the ions and $C_{S}$ represents the integral capacitance of the Stern layer.

\subsection{Validation of the model}

With the aim of checking the validity of our model, to show the significance of the Stern layer, and to demonstrate specific integral capacities, we extracted the sensitivity parameters and potential responses versus the $\mathrm{pHB}$ curve for the structures reported in Figure 2, and adapted experimental real data for a comparison of results [9].

Figure 3(a) (Gouy-Chapman) shows the sensitivity parameters of Sio2 as a function of pHB at an 0.1 electrolyte concentration in the Gouy-Chapman model. As shown, the maximum surface potential sensitivity lies between $\mathrm{pH} 6.5$ to $\mathrm{pH}$ 8. The lowest sensitivity up to zero charge is approximately $(\mathrm{pH}=2)$ due to a low Bint. As shown, perhaps the most serious disadvantage of this model is the curve roll-off when the $\mathrm{pH}$ becomes higher than $\mathrm{pH}=10$. In contrast to earlier findings, however, no roll-off of the Stern model was detected, as shown in Figure 3(a). These findings will doubtless be much scrutinised, but there are some immediately dependable conclusions for total sensitivity as shown in Figure 3b. What can be clearly seen in this chart is the high rate of sensitivity achieved in the Stern model - closer to the Nernst level. Figure 4 is a comparison of the experimental real data with the theoretical response, using the two models for Sio2. The sensitivity from experimental data is slightly higher than the theoretical sensitivity results. A GouyChapman-Stern model with 0.2 Cs achieved a better fit with real data. 


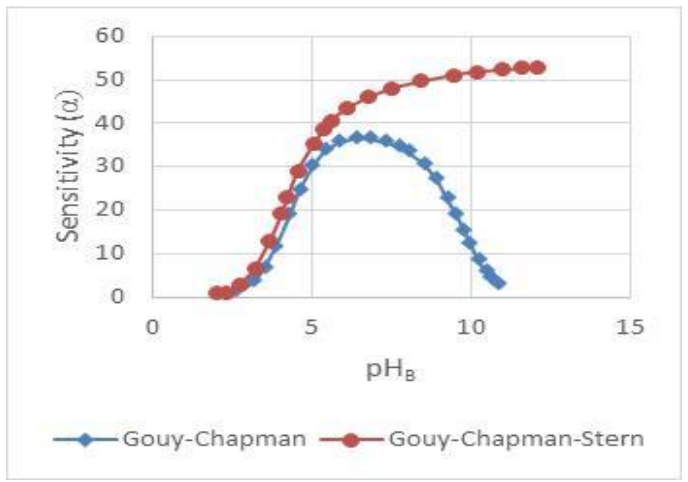

(a)

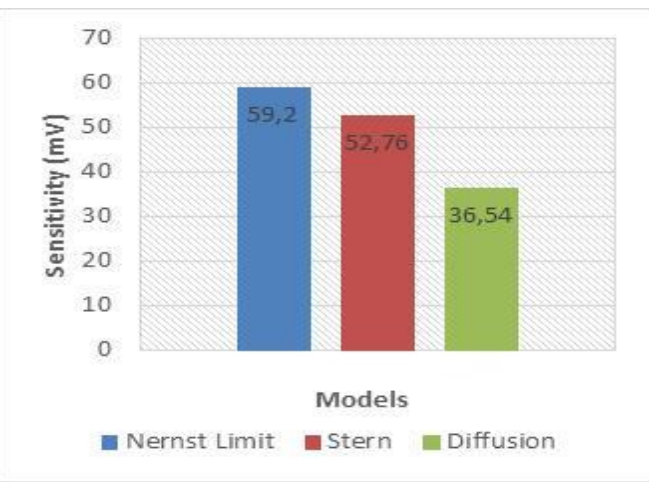

(b)

Figure 3. Theoretical and experimental sensitivities of ISFET: (a) $\mathrm{Sio}_{2}$ sensitivity parameters for two models, and (b) Total sensitivity models comparing with Nernst limit

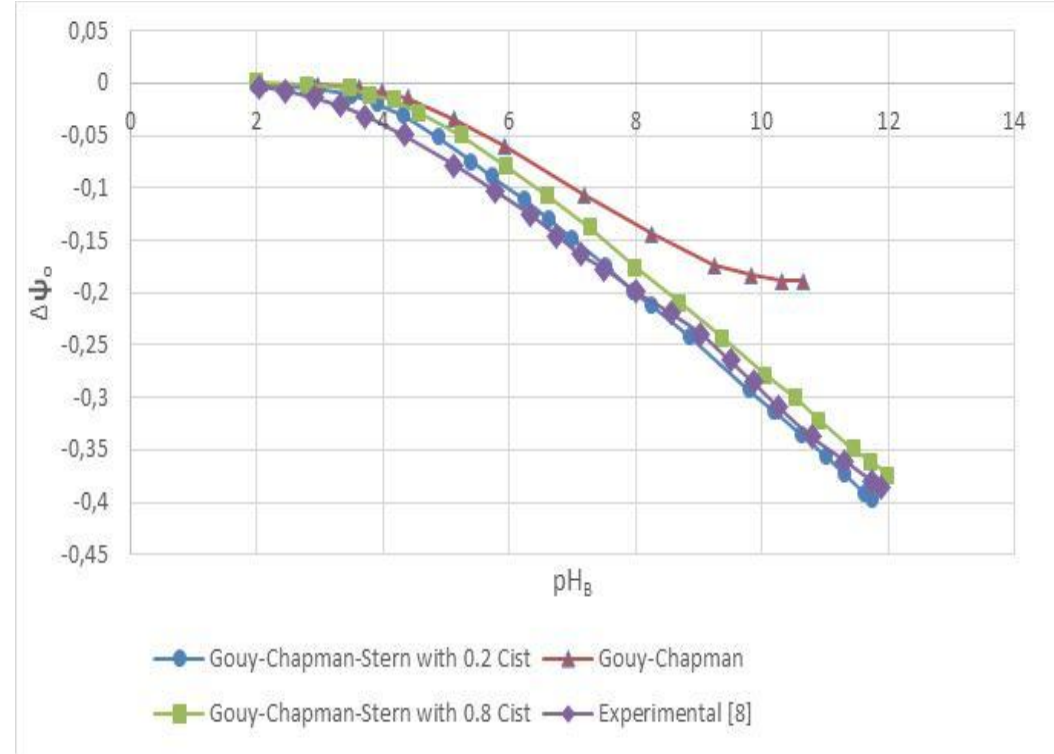

Figure 4. Theoretical and experimental total responses of a $\mathrm{SiO} 2$

From the previous validation, we can conclude that the Stern layer with a specific end goal provide the significance to replicate the measurements at high $\mathrm{pH}$. The reason is that when the cations collect at the base separation from the dielectric/electrolyte interface, a voltage drop is created over the Stern layer. Therefore, specifically in high $\mathrm{pH}$ bulk, instead of using a Stern layer, electrolyte ions will cover the surface charge causing threshold voltage weakness and a roll-off when the $\mathrm{pH}$ is higher than $\mathrm{pH}=10$.

Regarding previous validation and the conclusions from our adapted model, the next part of this paper presents a comprehensive comparison of five common materials in use in microelectronic applications (A12O3, Ta2O5, Hfo2, Zro2 and SN2O3) using the same method.

\section{RESULTS AND DISCUSSION}

The previous section showed that the Stern model affords excellent agreement with experimental data. Moreover, this model showed the highest and most stable sensitivity parameters. Therefore, in this section, we report a comparison of five materials common in microelectronic applications (A12O3, Ta2O5, Hfo2, Zro2 and SN2O3).

An initial objective of the project was to identify a high pH-sensitivity insulator layer (Stern Layer) material based on the surface potential sensitivity parameter. One interesting finding was examining the 
influence of the surface potential to $\mathrm{pH}$ bulk change. Figure 5 shows that using the adapted model to calculate the theoretical surface potential of (Al2O3, Ta2O5, Hfo2, Zro2 and SN2O3) as a function of pH bulk. The two materials that cover a large space of surface potential are Ta2O5 and Zro2. What can be clearly seen in this chart is the high rate of response of surface potential when using Tantalum material (Ta2O5). Ta2O5 rose to a high point and peaked at approximately $0.52 \mathrm{~V}$ compared with other materials. $\mathrm{Al} 2 \mathrm{O} 3$ and $\mathrm{Hfo} 2$ revealed a stable surface response to $\mathrm{pH}$ bulk change.

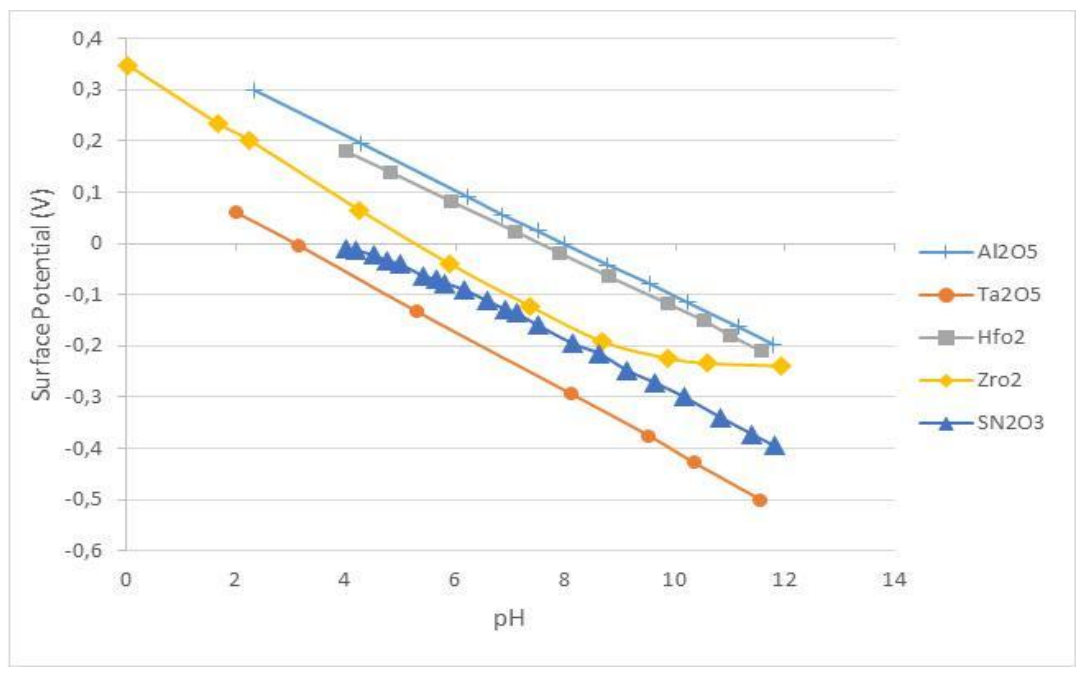

Figure 5. Interface surface potential versus $\mathrm{pH}$ relationship

These curves would have been more useful if they had focused on the pH sensitivity value as one of the most important values. These values can remove doubt about which material can achieve the highest sensitivity. Therefore, the calculations of $\mathrm{pH}$-change sensitivity of the five previous high-k materials have been reported based on the adapted model as shown in Figure 6. For more validation, real experimental data gathered from existing literature was compared with theoretical calculations. The experimental data for $\mathrm{A} 12 \mathrm{O} 3$ and Ta2O5 was collected from [9], Hfo2 from [15], Zro2 from [12] and SN2O3 from [10]. The results in Figure 5 are consistent with the data obtained in Figure 4, and confirms that Ta2O5 rose to a high point and peaked at approximately $59 \mathrm{mV} / \mathrm{pH}$, within easy reach of the ideal sensitivity (Nernst Limit). Zro2 tended towards the next high sensitivity value at around $56 \mathrm{mV} / \mathrm{pH}$. Al2O3 and Hfo2 satisfied the same rate at approximately $52 \mathrm{mV} / \mathrm{pH}$. The last one is $\mathrm{SN} 2 \mathrm{O} 3$, which theoretically reached around $46 \mathrm{mV} / \mathrm{pH}$. Obviously, these results support previous research into this topic.

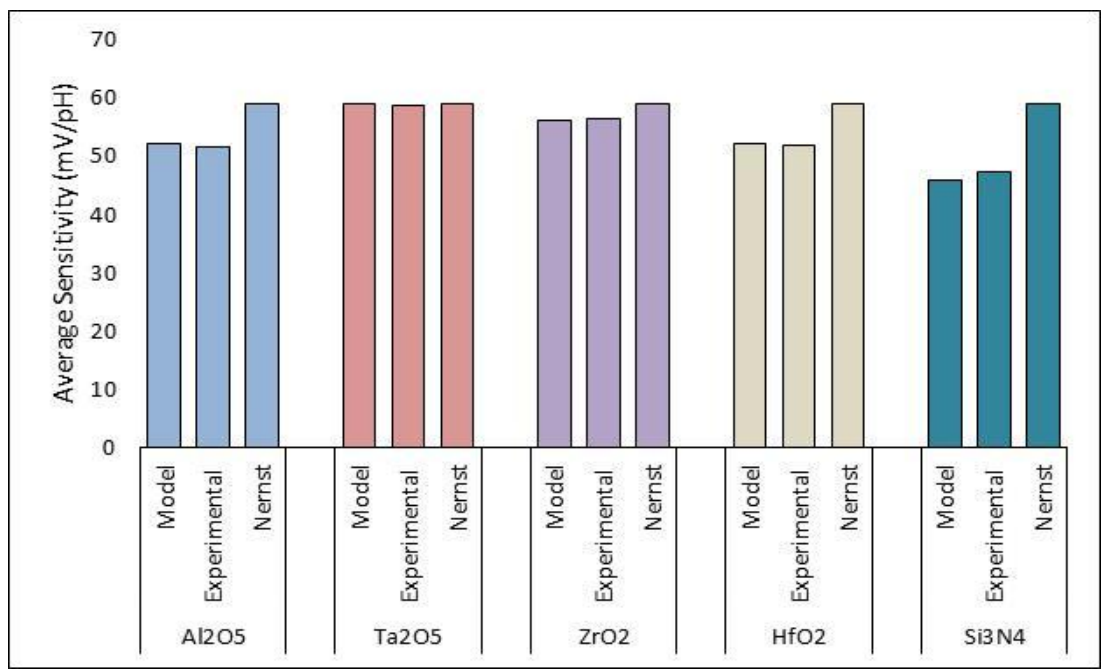

Figure 6. Comparison of the theoretical sensitivities 
A comparison of the two results reveals that Ta2O5 exhibits the best potential for an ISFET Stern layer. The last step in these findings is to examine the surface potential stability of Ta2O5 in terms of the electrolyte concentration change, using Equation (9). Three electrolyte concentrations, $10 \mathrm{mM}, 100 \mathrm{mM}$ and $1 \mathrm{mM}$, were used. The results, as shown in Figure 7 , indicate that the response of surface potential as calculated by the ISFET model is a little impact by the ionic and electrolyte for different concentrations.

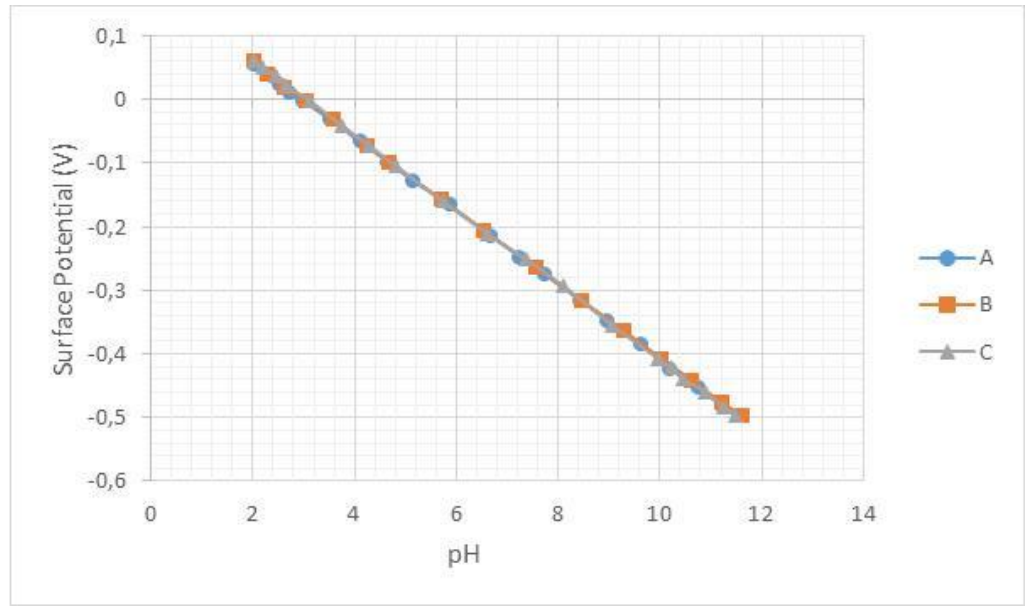

Figure 7. Surface potential of $\mathrm{Ta}_{2} \mathrm{O}_{5}$ ISFET in (A) $100 \mathrm{mM}$, (B) $10 \mathrm{mM}$ and (C) $1 \mathrm{mM}$

\section{CONCLUSION}

This study set out to identify different high-k materials to use as sensing membranes, rather than using SiO2. The Gouy-Chapman and Gouy-Chapman-Stern models were combined with the Site-binding model, based on surface potential sensitivity. In summary, the results showed that Ta2O5 has a high surface potential sensitivity and exhibits high stability in different electrolyte concentrations. The findings of this research provide insights for examining different materials before going on to expensive real ISFET fabrication. Further research needs to examine more closely the links between IC design simulation and these models for more accurate analysis and further optimisation.

\section{ACKNOWLEDGEMENT}

The authors would like to take this opportunity to highly appreciate the cooperation and the opportunity given by UTeM organization and UTeM Zamalah for funding this research. And the opportunity given by RACE Grant: RACE/F3/TK3/FKEKK/F00299.

\section{REFERENCES:}

[1] A. Bratov, N. Abramova, and C. Domínguez, "Investigation of chloride sensitive ISFETs with different membrane compositions suitable for medical applications," Anal. Chim. Acta, vol. 514, no. 1, pp. 99-106, 2004.

[2] C. S. Lee, S. Kyu Kim, and M. Kim, "Ion-sensitive field-effect transistor for biological sensing," Sensors, vol. 9, no. 9, pp. 7111-7131, 2009.

[3] T. Sakurai and Y. Husimi, "Real-Time Monitoring of DNA Polymerase Reactions by a Micro ISFET pH Sensor," Anal. Chem., vol. 64, no. 17, pp. 1996-1997, 1992.

[4] A. M. Dinar, A. S. M. Zain, and F. Salehuddin, "CMOS ISFET device for DNA Sequencing: Device Compensation, Application Requirements and Recommendations," vol. 12, no. 21, pp. 11015-11028, 2017.

[5] A. M. Dinar, A. S. M. Zain, and F. Salehuddin, "Utilizing of Cmos Isfet Sensors in Dna Applications Detection : A Systematic Review", vol. 10, pp. 569-583, 2018.

[6] P. Bergveld, "Development, operation, and application of the ion-sensitive field-effect transistor as a tool for electrophysiology.," IEEE Trans. Biomed. Eng., vol. 19, no. 5, pp. 342-351, 1972.

[7] M.-N. Niu, X.-F. Ding, and Q.-Y. Tong, "Effect of two types of surface sites on the characteristics of Si3N4-gate pH-ISFETs," Sensors Actuators B Chem., vol. 37, no. 1-2, pp. 13-17, 1996.

[8] S. Chen, J. G. Bomer, E. T. Carlen, and A. Van Den Berg, "A12O3/silicon nanoISFET with near ideal nernstian response," Nano Lett., vol. 11, no. 6, pp. 2334-2341, 2011.

[9] R. E. G. Van Hal, J. C. T. Eijkel, and P. Bergveld, "A general model to describe the electrostatic potential at electrolyte oxide interfaces,” Adv. Colloid Interface Sci., vol. 69, no. 1-3, pp. 31-62, 1996. 
[10] D. L. Harame, L. J. Bousse, J. D. Shott, and J. D. Meindl, "Ion-Sensing Devices with Silicon Nitride and Borosilicate Grlass Insulators," IEEE Trans. Electron Devices, vol. 34, no. 8, pp. 1700-1707, 1987.

[11] D.-H. Kwon, B.-W. Cho, C.-S. Kim, and B.-K. Sohn, "Effects of heat treatment on Ta2O5 sensing membrane for low drift and high sensitivity pH-ISFET," Sensors Actuators B Chem., vol. 34, no. 1-3, pp. 441-445, 1996.

[12] T. Akiyama, Y. Ujihira, Y. Okabe, T. Sugano, and E. Niki, "Ion-Sensitive Field-Effect Transistors with Inorganic Gate Oxide for pH Sensing,” IEEE Trans. Electron Devices, vol. 29, no. 12, pp. 1936-1941, 1982.

[13] H. R. Thakur, G. Keshwani, J. C. Dutta, and C. Engineering, "Sensitivity of Carbon Nanotube Based Junctionless Ion Sensitive Field Effect Transistor (CNTJLISFET) for $\mathrm{HfO} 2$ and $\mathrm{ZrO} 2$ gate dielectrics: Experimental and Theoretical Investigation," pp. 137-142, 2017.

[14] V. Jankovic and J. P. Chang, "HfO2 and ZrO2-Based Microchemical Ion Sensitive Field Effect Transistor (ISFET) Sensors: Simulation \& Experiment," J. Electrochem. Soc., vol. 158, no. 10, p. P115, 2011.

[15] A. Tarasov et al., "Understanding the electrolyte background for biochemical sensing with ion-sensitive field-effect transistors," ACS Nano, vol. 6, no. 10, pp. 9291-9298, 2012.

[16] W. Huang, A. K. Diallo, J. L. Dailey, K. Besar, and H. E. Katz, "Electrochemical processes and mechanistic aspects of field-effect sensors for biomolecules," J. Mater. Chem. C, vol. 3, no. 25, pp. 6445-6470, 2015.

[17] P. Bergveld, "Short Communications: Development of an Ion-Sensitive Solid-State Device for Neurophysiological Measurements," IEEE Trans. Biomed. Eng., vol. BME-17, no. 1, pp. 70-71, 1970.

[18] P. Bergveld, "Thirty years of ISFETOLOGY," Sensors Actuators B Chem., vol. 88, no. 1, pp. 1-20, 2003.

[19] M. W. S. R. S. Cobbold, "Basic Properties of the Electrolyte-Si02-Si System :," IEEE Trans. Electron Devices, no. 1, pp. 1805-1815, 1979.

[20] D. M. Wilson, S. Hoyt, J. Janata, K. Booksh, and L. Obando, "Chemical sensors for portable, handheld field instruments," IEEE Sens. J., vol. 1, no. 4, pp. 256-274, 2001.

[21] P. Kurzweil, Metal oxides and ion-exchanging surfaces as $\mathrm{pH}$ sensors in liquids: State-of-the-art and outlook, vol. 9, no. 6. 2009.

[22] B. Chen, A. Parashar, and S. Pandey, "Folded floating-gate CMOS biosensor for the detection of charged biochemical molecules," IEEE Sens. J., vol. 11, no. 11, pp. 2906-2910, 2011.

[23] L. L. Chi, J. C. Chou, W. Y. Chung, T. P. Sun, and S. K. Hsiung, "Study on extended gate field effect transistor with tin oxide sensing membrane," Mater. Chem. Phys., vol. 63, no. 1, pp. 19-23, 2000.

[24] W. Healy, D. E. Yates, and S. Levine, "Site-binding Model of the Electrical Double Layer at the Oxide / Water Interface," Trans. Farad. Soc. I, vol. 70, p. 1807, 1974.

[25] H. K. Jung, "Projected range dependent tunneling current of asymmetric double gate MOSFET," Int. J. Electr. Comput. Eng., vol. 6, no. 1, pp. 113-119, 2016.

[26] H. K. Jung and S. Dimitrijev, "The impact of tunneling on the subthreshold swing in Sub-20 nm asymmetric double gate MOSFETs," Int. J. Electr. Comput. Eng., vol. 6, no. 6, pp. 2730-2734, 2016.

\section{BIOGRAPHIES OF AUTHORS}
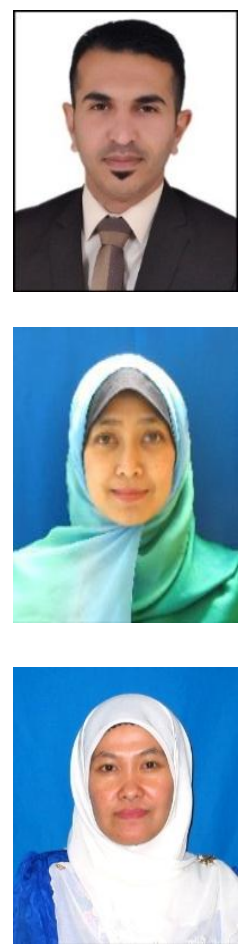

Ahmed M. Dinar was born in Baghdad, Iraq in 1984. He received his B. Eng. degree computer engineering from college of Engineering/ Al-Mustanairiay University in 2007. He received M. Sc degree from Computer Engineering department/Uneversity of Technology/Iraq in 2014. Currently, $\mathrm{He}$ is a Ph.D. student at Faculty of Electronic and Computer Engineering (FKEKK), Universiti Teknikal Malaysia Melaka since 2017 where his Ph.D. research focuses on CMOS/ISFET sensor for Cancer Detection. he is currently a Lecturer with computer engineering department,University of Technology, Baghdad, Iraq.

A. S. Mohd Zain was graduated B. Eng degree in Electric, Electronic \& System from the University Kebangsaan Malaysia. She received the M.Sc. degree in Microelectronics from University Kebangsaan Malaysia and Ph.D. degree in Microelectronics from University of Glasgow United Kingdom. She is a Senior Lecturer at Universiti Teknikal Malaysia Melaka. Her research interests include IC Design for Biomedical Applications and Nanoscale Device Design and Simulation.

F. Salehuddin was received B. Eng degree in communication engineering from the MARA University Of Technology. She received the M.Sc. degree in Microelectronics from University Kebangsaan Malaysia and Ph.D. degree in Microelectronics from Universiti Tenaga Nasional, Malaysia. She is a Senior Lecturer at Universiti Teknikal Malaysia Melaka. Her research interests include Device and Process Simulation of CMOS, Optimization Approach (DOE) and Variability of nanoscale device. 